\title{
Abcès cervical isolé : une cause rare de torticolis fébrile chez l'adulte
}

\author{
Isolate Cervical Abcess an Uncommon Cause of Stiff Neck Associated with Fever in Adults
}

\section{A. Jusseaume · E. Kras}

Reçu le 28 novembre 2014; accepté le 2 avril 2015

(C) SFMU et Lavoisier SAS 2015

Une femme de 58 ans aux antécédents de cancer du sein en rémission se présente aux urgences pour un torticolis dans un contexte d'hyperthermie évoluant depuis 72 heures. L'interrogatoire ne retrouve pas de traumatisme cervical récent ni de contage infectieux. La température est à $38,5^{\circ} \mathrm{C}$. L'examen clinique retrouve une contracture du muscle sternocléido-mastoïdien droit. Le reste de l'examen, notamment ORL, est sans particularité (pas d'angine, pas de phlegmon pharyngé, pas de porte d'entrée cutanée locale). La biologie retrouve une CRP à $247 \mathrm{mg} / \mathrm{L}$, une hyperleucocytose à 14,62 G/L (11,7 G/L de polynucléaires neutrophiles).

A. Jusseaume $\cdot$ E. Kras $(\bowtie)$

Actes Urgences, 6 avenue des peupliers,

F-13960 Sausset-les-Pins

e-mail : etiennekr@hotmail.com
Devant l'absence de point d'appel infectieux retrouvé cliniquement et la disponibilité de l'IRM à ce moment de la prise en charge, une IRM cervicale est effectuée aux urgences. Elle retrouve un abcès de $12 \mathrm{~mm}$ des parties molles cervicales avec une zone inflammatoire au contact des vertèbres C3, C4, sans atteinte des structures osseuses ou discoligamentaires (Figs 1A, 1B). L'évolution a été favorable après antibiothérapie instaurée aux urgences et drainage chirurgical dans les 24 heures. Le torticolis fébrile de l'adulte est plus rare que chez l'enfant. Les étiologies les plus fréquentes sont une infection des espaces para- et rétropharyngés, une spondylodiscite/arthrite septique ou une méningite de présentation atypique. L'abcès des parties molles cervical isolé est une autre cause rare de torticolis fébrile chez l'adulte, l'apport de l'IRM aux urgences permet le diagnostic et l'initiation d'une thérapeutique médicochirurgicale précoce. 